\title{
Perioperative lung protection
}

\author{
Korean Journal of Anesthesiology Katherine Marseu and Peter Slinger \\ Department of Anesthesia and Pain Management, Toronto General Hospital, University of Toronto, Toronto, \\ Canada
}

Perioperative pulmonary complications are known to be a major cause of morbidity and mortality, and as such, contribute a large burden to the health care system globally. Anesthesiologists have an important role during the perioperative period to identify patients at risk of these complications and intervene in order to reduce them. After describing perioperative pulmonary complications and risk factors for such, this article will address preoperative, intraoperative, and postoperative lung protective strategies to try and reduce the risk of these complications.

Key Words: Lung protection, Perioperative pulmonary complications.

\section{Perioperative Pulmonary Complications}

Pulmonary complications have been shown to be a major cause of morbidity and mortality in the perioperative period, in both cardiothoracic surgical populations, and non-cardiothoracic surgery [1-5]. Several large studies that have looked at perioperative pulmonary complications (PCC) include a 2000 prospective cohort study of over 80,000 American veterans [6], a 2006 systematic review by the American College of Physicians (ACP) [2,3], and a 2011 multi-center, European observational study [7]. However, there are some inconsistent findings between these studies. Factors that make it difficult to study PCC include the disparate populations used, the wide variety of surgical procedures involved, and the large list of potential pulmonary complications to consider. PCC can encompass a wide variety of pathologies, such as exacerbation of chronic lung disease, pneumothorax, pulmonary embolism, pulmonary edema, atelectasis, pneumonia, acute lung injury (ALI), acute respira-

Corresponding author: Peter Slinger, M.D.

Department of Anesthesia and Pain Management, University Health Network - Toronto General Hospital, 200 Elizabeth Street, Eaton North, 3rd Floor, Room 437, Toronto, Ontario M5G 2C4, Canada Tel: 416-340-5164, Fax: 416-340-3698

Email: peter.slinger@uhn.ca

Received: April 25, 2017.

Accepted: May 4, 2017.

Korean J Anesthesiol 2017 June 70(3): 239-244

https://doi.org/10.4097/kjae.2017.70.3.239 tory distress syndrome (ARDS), and respiratory failure [2,4,5], the latter of which can be fatal in up to $20 \%$ of patients within 30 days following surgery [4]. In the thoracic surgical population specifically, PCC occur in up to $20 \%$ of patients and account for most of the expected 5\% mortality in this population [8]. In non-cardiothoracic surgery, there has been less research into PCC compared to cardiac complications, even though PCC have been shown to occur with a similar, if not higher, frequency, depending on the study [2-4,9]. In fact, in a retrospective cohort study of over 45,000 patients undergoing bowel surgery, Fleisher and Linde-Zwirble [10] found a 19\% rate of PCC compared to just a $1.2 \%$ rate of cardiac complications, with the PCC costing almost 3.5\% more.

It is clear that PCC have the potential to place a large burden on health care systems. Previous studies have focused mainly on North American and European populations [2,3,6,7], as have reviews on this subject, including a previous review by the authors of this paper [11]. This present review will attempt to amalgamate evidence from the North American and European populations with that from others areas of the world. It is important to extend findings on PCC to other geographic areas and populations globally, so that a greater number of anesthesiologists may be able to better determine which patients will be at risk for them, and intervene in order to reduce this risk.

\section{Risk Factors for Perioperative Pulmonary Complications}

Conditions associated with an increased risk of PCC can be

(c) This is an open-access article distributed under the terms of the Creative Commons Attribution Non-Commercial License (http://creativecommons.org/ licenses/by-nc/4.0/), which permits unrestricted non-commercial use, distribution, and reproduction in any medium, provided the original work is properly cited. 
classified into patient-related factors and procedure-related factors. According to Arozullah and colleagues and the ACP clinical guidelines for non-cardiothoracic surgery, patient-related factors have been shown to include advanced age, impaired health and functional status, substance use, and associated comorbidities [1,2,4-6,12-14]. Age greater than 60 years old is associated with higher risk of PCC, but it is unclear if this is due solely to biological age, or to the likely association of increased comorbidities $[1,12]$. An increased American Society of Anesthesiologists (ASA) score may be related to poor health and functional status $[1,2,4]$. Traditionally, hypoalbuminemia $(<30-35 \mathrm{~g} / \mathrm{L})$ has been a significant factor associated with PCC $[2,6]$, and this may also be due to its relationship to poor health and functional status. The use of substances such as alcohol and cigarettes conveys moderately increased risk of PCC according to the ACP guidelines $[1,2,4,12]$. Comorbidities such as congestive heart failure, renal insufficiency, and chronic obstructive pulmonary disease (COPD) are most likely to increase the risk of PCC [1,2,5,6,12]. Impaired spirometric values of lung function in patients with chronic lung disease undergoing non-thoracic surgery do not seem to correlate with an increased risk of PCC [2,7]. Risk factors for postoperative respiratory failure in the thoracic surgical population specifically are similar to the general population, and also include decreased preoperative respiratory function, as denoted by the forced expiratory volume in 1 second, extent of planned lung resection, and the presence of coronary artery disease [15].

Additional patient factors that have more recently been shown to contribute to pulmonary risk include obstructive sleep apnea (OSA), obesity hypoventilation syndrome (OHS) and pulmonary hypertension $[1,4,12,14]$. Patients with OSA have been found to have higher rates of postoperative hypoxia, aspiration pneumonia, reintubation and hospital length of stay than those without OSA [14]. OHS is characterized by chronic hypercapnia $\left(\mathrm{PaCO}_{2}>45 \mathrm{mmHg}\right.$ ), sleep disordered breathing, and a body mass index $>30 \mathrm{~g} / \mathrm{m}^{2}$. The severity of illness is under-recognized in most of these patients undergoing elective surgery, which places them at even higher risk of postoperative respiratory failure than those with OSA [14]. Both OSA and OHS may contribute to pulmonary hypertension and right-heart dysfunction. Patients with this disease are at very high risk of respiratory failure, mechanical ventilation, and prolonged intensive care unit length of stay [14]. Interestingly, obesity and asthma, provided it is well controlled, have not been shown to be risk factors for PCC $[1,2,4,5,12]$.

Procedure-related factors may be more important than patientrelated factors in conveying risk, and include duration, type of surgical procedure, and anesthetic technique [1,2,4-6,12]. Prolonged surgery greater than three hours is associated with increased risk of PCC [1]. Surgical procedures near the diaphragm, such as aortic, thoracic, and upper abdominal carry a high risk of pulmonary complications, even in healthy patients [4]. This is likely due to diaphragmatic dysfunction and resultant low lung volumes, atelectasis, hypoxia, and subsequent respiratory compromise [5]. Laparoscopic compared to open abdominal surgeries improve pain scores, oxygenation, and lung function based on spirometry. According to the ACP review, though, it is unclear whether this translates into a clinically significant reduction in PCC [3]. However, a 2008 analysis of over 19,000 patients undergoing bariatric surgery showed that PCC were cut in half if patients underwent laparoscopic instead of open surgery [4]. Additionally, neurosurgery, head and neck surgery, and emergency surgery are also considered high-risk procedures. General anesthesia carries a higher risk for PCC than other anesthetic techniques, such as neuraxial anesthesia and peripheral nerve block. This is due to a number of factors: the disruption of the central regulation of breathing; attenuation of respiratory muscle activity; decreased functional residual capacity; hypoventilation; positional dependence; the development of atelectasis, and thus, impaired gas exchange. Residual neuromuscular blockade may also be implicated here [2-4,9].

With this discussion of risk factors for PCC in hand, we will now focus on potential preoperative, intraoperative, and postoperative interventions that may offer protection against PCC and thus decrease their incidence.

\section{Preoperative Lung Protective Strategies}

Several preoperative interventions may reduce PCC, including optimization of respiratory status, smoking cessation, and physiotherapy/rehabilitation [3].

Patients with chronic respiratory diseases, such as COPD and asthma, should be at their baseline prior to elective surgery $[1,6,9]$. Communication with primary care physicians or respirologists can be helpful to determine level of optimization. According to a Japanese review of guideline-oriented management, this includes control of airway hyperresponsiveness and infection, and the aggressive use of anticholinergics in COPD and beta- 2 adrenergic stimulants in asthma, as well as steroids, to limit acute exacerbations. This review also found that preoperative control with leukotriene antagonists can reduce the incidence of PCC [16].

Preoperative smoking cessation is an interesting matter. According to the Guidelines on Perioperative Smoking Cessation from the Safety Committee of the Japanese Society of Anesthesiologists, smoking increases perioperative complications resulting in delayed postoperative recovery, and it is meaningful to stop smoking at any point in the preoperative period [17]. The 2006 ACP review found that cessation may actually increase short-term pulmonary risk because of increased mucus produc- 
tion from improved mucociliary function and decreased coughing from less bronchial irritation [3]. However, in the cardiothoracic surgical population, longer periods of smoking cessation have been found to lead to greater risk-reduction for PCC. A period of at least four to eight weeks of cessation is recommended in order to decrease PCC, including ARDS [18,19]. Even at 12-48 hours post cessation, carboxyhemoglobin concentrations decrease. Wound healing is also improved in patients who stop smoking more than four weeks pre-operatively, as smoking leads to a prolonged period of tissue hypoxemia. Cessation for 2-3 months will lead to decreased sputum production $[9,19]$. Though smoking cessation should always be encouraged, the best timing for this with respect to surgery is still controversial [6].

The perioperative risk profile in patients with poor functional capacity may be improved in those who undergo preoperative rehabilitation, or "pre-habilitation," which includes comprehensive programs of physical exercise, chest physiotherapy, and optimization of nutritional status [10,20]. Programs such as these increase exercise ability, as impaired functional capacity is associated with postoperative complications and all-cause mortality in major surgery $[18,21]$. In patients with COPD, preoperative exercise programs are known to decrease the risk of PCC in the setting of lung volume reduction surgery, lung cancer surgery and lung transplantation [20,22]. However, several studies have found no difference in outcomes after other major surgeries in patients participating in preoperative exercise programs $[21,23]$. Preoperative chest physiotherapy that incorporates lung expansion techniques, such as active breathing, incentive spirometry, and forced expiration techniques, were found to be associated with 50-60\% less PCC, including pneumonia, in patients who performed these exercise for 20 minutes a day for at least two weeks prior to surgery $[4,11]$. A recent systematic review also confirmed a 50\% reduction in PCC in patients undergoing cardiothoracic and upper abdominal surgery who completed preoperative respiratory muscle training programs. It is thought that respiratory muscle weakness contributes to PCC [24].

\section{Intraoperative Lung Protective Strategies}

Intraoperative interventions that may reduce PCC include lung protective mechanical ventilation, judicious fluid administration, and adequate analgesia. The use of volatile anesthetics in lung protection will be briefly discussed in this section as well.

Historically, large tidal volumes up to $15 \mathrm{ml} / \mathrm{kg}$ were used in the perioperative period in order to avoid atelectasis. However, given the work done in ARDS management, mechanical ventilation is now known to be associated with inflammation and cell injury. Markers of these processes have been found to be elevated in patients ventilated with larger tidal volumes. Thus, the current trend in mechanical ventilation is to use tidal volumes of approximately $6 \mathrm{ml} / \mathrm{kg}$, which is a normal spontaneous tidal volume. Volumes larger than this have been shown to be a major risk factor for the development of ALI in mechanically ventilated patients (so-called ventilator induced lung injury or VILI) [25]. A prospective study found that tidal volumes $>700$ $\mathrm{ml}$ and peak airway pressure $>30 \mathrm{cmH}_{2} \mathrm{O}$ were independently associated with the development of ARDS [26]. It is important to remember that the majority of these findings have even been shown in patients with previously healthy lungs. ALI is the most common cause of postoperative respiratory failure and may be associated with mortality rates as high as $45 \%$ [27]. Though good quality randomized-controlled trials looking specifically at the interaction between perioperative mechanical ventilation strategies, ALI and PCC are lacking, it would seem reasonable to apply the lung protective strategies learned from ARDS management to the operative period.

Fluid management has always been a controversial issue. Excessive intraoperative fluid resuscitation and transfusion have been shown to be risk factors for the development of perioperative ALI and ARDS $[27,28]$. This is especially true in cardiothoracic surgery, where fluid overload may add to the pulmonary endothelial damage already caused by the inflammatory reaction from mechanical ventilation and cardiopulmonary bypass [29-31]. This leads to capillary damage and pulmonary edema [32]. In a 2012 meta-analysis of major surgeries, liberal fluid management was associated with a higher risk of pulmonary edema, pneumonia, and hospital length of stay [33]. On the other hand, a concern with restrictive fluid management is the possible contribution to decreased organ perfusion and dysfunction, particularly the kidneys, as well as decreased perfusion of the surgical anastamosis [31]. The current trend in ideal fluid management for major surgeries is individualized to optimize cardiac output and oxygen delivery, while avoiding excessive fluid administration. Hemodynamic endpoints such as stroke volume, cardiac output, and measures of fluid responsiveness such as pulse pressure and stroke volume variation (collectively referred to as goal directed fluid therapy) may provide a superior alternative to fixed regimens [31].

Neuraxial analgesia may contribute to less pain-related hypoventilation and respiratory muscle dysfunction in highrisk abdominal, esophageal, aortic, and cardiac surgery [12]. Evidence was conflicting, though, in the ACP review of noncardiothoracic surgery as to whether this consistently translated into less PCC [3]. However, a 2007 systematic review went on to show that thoracic epidural analgesia (TEA) reduced PCC rates by one-third to one-half in aortic, cardiac, and abdominal surgery [12]. This may be explained by earlier extubation, improved analgesia during mobilization and coughing, reduction of bronchial reactivity, and improved diaphragmatic function [34]. 
TEA has been considered the gold standard for post-operative pain control in patients undergoing thoracotomy. It has been shown that when thoracotomy pain is controlled and patients are able to cough, there is less respiratory dysfunction and complication [34]. In patients with coronary artery disease, thoracic epidural local anesthetics can also reduce myocardial oxygen demand. Likewise, consideration should be given to using neuraxial anesthesia and/or peripheral nerve blocks as the primary method of anesthesia where possible, and especially in patients with OSA, to avoid the decrease in lung volumes that occur with general anesthesia [14]. These changes persist postoperatively and are compounded by pain [3].

Volatile anesthetics are known to have immune-modulatory properties by inhibiting the expression of pro-inflammatory mediators. Though early work in this area focused on the role of volatile anesthetics in cardiac surgery, more recently, they have also been shown to pre-condition and post-condition against ischemia-reperfusion injury in the lungs, as well as ALI [35-38]. Several studies in the thoracic surgical population undergoing one-lung ventilation have shown that volatile anesthesia compared to propofol anesthesia attenuates the inflammatory reaction, and even lowers adverse events and improves outcomes $[38,39]$. Though much work remains to be done in this area, current evidence suggests that volatile anesthetics may have a role in lung protection against the inflammatory response.

\section{Postoperative Lung Protective Strategies}

As alluded to in several of the preceding sections, decreased lung volumes and atelectasis occur in the perioperative period for a number of different reasons: surgical trauma causing diaphragmatic dysfunction; anesthetic related attenuation of respiratory muscle function; pain; positional dependence; and impaired mucociliary clearance [1]. Whatever the reason, decreased lung volumes and atelectasis are known to be associated with pulmonary complications [1]. Thus, postoperative interventions that focus on lung expansion techniques have been shown to reduce PCC by up to $50 \%$, especially in upper abdominal and thoracic surgery. These strategies include respiratory physiotherapy, incentive spirometry, and non-invasive ventilation (NIV). According to the 2006 ACP review, for patients having abdominal surgery, any of these lung expansion techniques were better than no intervention in reducing PCC [2-4,9].

Respiratory physiotherapy can include deep breathing, cough, postural drainage, percussion, vibration, suctioning, and ambulation. Incentive spirometry is an easy and inexpensive way to encourage deep breathing [2-4]. A prospective observational study of Australian patients undergoing upper abdominal surgery developed a clinical rule for predicting the risk of postoperative pulmonary complications from five patients and procedure related risk factors, including duration of anesthesia, type of surgery, smoking status, respiratory comorbidities, and exercise capacity measured by maximal oxygen uptake. The study concluded that these risk factors may be helpful in prioritizing which patients should receive postoperative respiratory physiotherapy [40].

NIV may be useful in patients who are unable to participate in incentive spirometry or respiratory physiotherapy. Continued positive airway pressure is particularly useful in those who have OSA. Benefits of NIV compared to invasive ventilation include fewer complications, lower morbidity and mortality rates, shorter hospital length of stay, and reduced costs overall. Evidence of benefit has been established in patients undergoing major thoracoabdominal or cardiac surgery, those with hypercapneic respiratory failure due to COPD or deformities of the chest wall, cardiogenic pulmonary edema, and in weaning from invasive mechanical ventilation. NIV may be used in both prophylactic and therapeutic fashions [41].

The last intervention that will be discussed here is the use of extracorporeal lung support (ECLS) devices, which allow for ultra-protective lung ventilation. This use has increasingly become an end-stage option in operative and critical care patients with respiratory failure, in whom other respiratory therapies have failed to provide adequate oxygenation and ventilation. The use of ECLS allows for the delivery of very low tidal volumes ( $3 \mathrm{ml} /$ $\mathrm{kg}$ ), low airway pressures, and low respiratory rates. These ventilatory settings have the potential to prevent further lung injury by attenuating the inflammatory response, even when compared to a tidal volume of $6 \mathrm{ml} / \mathrm{kg}$, as recommended in ARDS management [42-44].

\section{Summary}

Pulmonary complications are one of the major causes of morbidity and mortality in the perioperative period. Anesthesiologists have the important role of being able to identify patients at risk preoperatively, and can intervene during the whole perioperative period to try and mitigate these risks. Some of these interventions still have a conflicting evidence base. Therefore, it is important to continue research into PCC in order to provide a truly evidence-based approach to management in this field around the globe. Until this time however, lessons learned from other areas, such as pre-habilitation and smoking cessation in lung cancer patients, and ARDS management in critical care patients, can be applied to all major surgery to try and provide the best clinical care to all patient populations. 


\section{References}

1. Smetana GW. Preoperative pulmonary evaluation: identifying and reducing risks for pulmonary complications. Cleve Clin J Med 2006; 73 Suppl 1: S36-41.

2. Qaseem A, Snow V, Fitterman N, Hornbake ER, Lawrence VA, Smetana GW, et al. Risk assessment for and strategies to reduce perioperative pulmonary complications for patients undergoing noncardiothoracic surgery: a guideline from the American College of Physicians. Ann Intern Med 2006; 144: 575-80.

3. Lawrence VA, Cornell JE, Smetana GW. Strategies to reduce postoperative pulmonary complications after noncardiothoracic surgery: systematic review for the American College of Physicians. Ann Intern Med 2006; 144: 596-608.

4. Smetana GW. Postoperative pulmonary complications: an update on risk assessment and reduction. Cleve Clin J Med 2009; 76 Suppl 4: S605 .

5. Yang CK, Teng A, Lee DY, Rose K. Pulmonary complications after major abdominal surgery: National Surgical Quality Improvement Program analysis. J Surg Res 2015; 198: 441-9.

6. Arozullah AM, Daley J, Henderson WG, Khuri SF. Multifactorial risk index for predicting postoperative respiratory failure in men after major noncardiac surgery. The National Veterans Administration Surgical Quality Improvement Program. Ann Surg 2000; 232: 242-53.

7. Canet J, Hardman J, Sabaté S, Langeron O, Abreu MG, Gallart L, et al. PERISCOPE study: predicting post-operative pulmonary complications in Europe. Eur J Anaesthesiol 2011; 28: 459-61.

8. Slinger P, Darling G. Preanesthetic assessment for thoracic surgery. In: Principles and Practice of Anesthesia for Thoracic Surgery. Edited by Slinger P: New York, Springer. 2011, pp 11-34.

9. Warner DO. Preventing postoperative pulmonary complications: the role of the anesthesiologist. Anesthesiology 2000; 92: 1467-72.

10. Fleisher LA, Linde-Zwirble WT. Incidence, outcome, and attributable resource use associated with pulmonary and cardiac complications after major small and large bowel procedures. Perioper Med (Lond) 2014; 3: 7.

11. Marseu K, Slinger P. Peri-operative pulmonary dysfunction and protection. Anaesthesia 2016; 71 Suppl 1: 46-50.

12. Smetana GW, Conde MV. Preoperative pulmonary update. Clin Geriatr Med 2008; 24: 607-24.

13. Licker M, Triponez F, Diaper J, Karenovics W, Bridevaux PO. Preoperative evaluation of lung cancer patients. Current Anesthesiology Reports 2014; 4: 124-34.

14. Bhateja P, Kaw R. Emerging risk factors and prevention of perioperative pulmonary complications. ScientificWorldJournal 2014; 2014: 546758.

15. Falcoz PE, Conti M, Brouchet L, Chocron S, Puyraveau M, Mercier M, et al. The Thoracic Surgery Scoring System (Thoracoscore): risk model for in-hospital death in 15,183 patients requiring thoracic surgery. J Thorac Cardiovasc Surg 2007; 133: 325-32.

16. Yamakage M, Iwasaki S, Namiki A. Guideline-oriented perioperative management of patients with bronchial asthma and chronic obstructive pulmonary disease. J Anesth 2008; 22: 412-28.

17. Safety Committee of Japanese Society of Anesthesiologists. A guideline for perioperative smoking cessation. J Anesth 2017; 31: 297-303.

18. Warner MA, Offord KP, Warner ME, Lennon RL, Conover MA, Jansson-Schumacher U. Role of preoperative cessation of smoking and other factors in postoperative pulmonary complications: a blinded prospective study of coronary artery bypass patients. Mayo Clin Proc 1989; 64: 609-16.

19. Vaporciyan AA, Merriman KW, Ece F, Roth JA, Smythe WR, Swisher SG, et al. Incidence of major pulmonary morbidity after pneumonectomy: association with timing of smoking cessation. Ann Thorac Surg 2002; 73: 420-5.

20. Nici L. Preoperative and postoperative pulmonary rehabilitation in lung cancer patients. Thorac Surg Clin 2008; 18: 39-43.

21. Gillis C, Li C, Lee L, Awasthi R, Augustin B, Gamsa A, et al. Prehabilitation versus rehabilitation: a randomized control trial in patients undergoing colorectal resection for cancer. Anesthesiology 2014; 121: 937-47.

22. Morano MT, Araújo AS, Nascimento FB, da Silva GF, Mesquita R, Pinto JS, et al. Preoperative pulmonary rehabilitation versus chest physical therapy in patients undergoing lung cancer resection: a pilot randomized controlled trial. Arch Phys Med Rehabil 2013; 94: 53-8.

23. Lemanu DP, Singh PP, MacCormick AD, Arroll B, Hill AG. Effect of preoperative exercise on cardiorespiratory function and recovery after surgery: a systematic review. World J Surg 2013; 37: 711-20.

24. Mans CM, Reeve JC, Elkins MR. Postoperative outcomes following preoperative inspiratory muscle training in patients undergoing cardiothoracic or upper abdominal surgery: a systematic review and meta analysis. Clin Rehabil 2015; 29: 426-38.

25. Slutsky AS, Ranieri VM. Mechanical ventilation: lessons from the ARDSNet trial. Respir Res 2000; 1: 73-7.

26. Gajic O, Frutos-Vivar F, Esteban A, Hubmayr RD, Anzueto A. Ventilator settings as a risk factor for acute respiratory distress syndrome in mechanically ventilated patients. Intensive Care Med 2005; 31: 922-6.

27. Fernández-Pérez ER, Sprung J, Afessa B, Warner DO, Vachon CM, Schroeder DR, et al. Intraoperative ventilator settings and acute lung injury after elective surgery: a nested case control study. Thorax 2009; 64: 121-7.

28. Hughes CG, Weavind L, Banerjee A, Mercaldo ND, Schildcrout JS, Pandharipande PP. Intraoperative risk factors for acute respiratory distress syndrome in critically ill patients. Anesth Analg 2010; 111: 464-7.

29. Vaporciyan AA, Rice D, Correa AM, Walsh G, Putnam JB, Swisher S, et al. Resection of advanced thoracic malignancies requiring cardiopulmonary bypass. Eur J Cardiothorac Surg 2002; 22: 47-52. 
30. Slinger P, Kilpatrick B. Perioperative lung protection strategies in cardiothoracic anesthesia: are they useful? Anesthesiol Clin 2012; 30: 60728.

31. Chau EH, Slinger P. Perioperative fluid management for pulmonary resection surgery and esophagectomy. Semin Cardiothorac Vasc Anesth 2014; 18: 36-44.

32. Assaad S, Popescu W, Perrino A. Fluid management in thoracic surgery. Curr Opin Anaesthesiol 2013; 26: 31-9.

33. Corcoran T, Rhodes JE, Clarke S, Myles PS, Ho KM. Perioperative fluid management strategies in major surgery: a stratified meta-analysis. Anesth Analg 2012; 114: 640-51.

34. Groeben H. Epidural anesthesia and pulmonary function. J Anesth 2006; 20: 290-9.

35. Fujinaga T, Nakamura T, Fukuse T, Chen F, Zhang J, Ueda S, et al. Isoflurane inhalation after circulatory arrest protects against warm ischemia reperfusion injury of the lungs. Transplantation 2006; 82: 1168-74.

36. Reutershan J, Chang D, Hayes JK, Ley K. Protective effects of isoflurane pretreatment in endotoxin-induced lung injury. Anesthesiology 2006; 104: 511-7.

37. Voigtsberger S, Lachmann RA, Leutert AC, Schläpfer M, Booy C, Reyes L, et al. Sevoflurane ameliorates gas exchange and attenuates lung damage in experimental lipopolysaccharide-induced lung injury. Anesthesiology 2009; 111: 1238-48.

38. Schilling T, Kozian A, Kretzschmar M, Huth C, Welte T, Bühling F, et al. Effects of propofol and desflurane anaesthesia on the alveolar inflammatory response to one-lung ventilation. Br J Anaesth 2007; 99: 368-75.

39. De Conno E, Steurer MP, Wittlinger M, Zalunardo MP, Weder W, Schneiter D, et al. Anesthetic-induced improvement of the inflammatory response to one-lung ventilation. Anesthesiology 2009; 110: 1316-26.

40. Scholes RL, Browning L, Sztendur EM, Denehy L. Duration of anaesthesia, type of surgery, respiratory co-morbidity, predicted VO2max and smoking predict postoperative pulmonary complications after upper abdominal surgery: an observational study. Aust J Physiother 2009; 55: 191-8.

41. Mathai AS. Non-invasive ventilation in the postoperative period: Is there a role? Indian J Anaesth 2011; 55: 325-33.

42. Frank JA, Gutierrez JA, Jones KD, Allen L, Dobbs L, Matthay MA. Low tidal volume reduces epithelial and endothelial injury in acidinjured rat lungs. Am J Respir Crit Care Med 2002; 165: 242-9.

43. Iglesias M, Jungebluth P, Petit C, Matute MP, Rovira I, Martínez E, et al. Extracorporeal lung membrane provides better lung protection than conventional treatment for severe postpneumonectomy noncardiogenic acute respiratory distress syndrome. J Thorac Cardiovasc Surg 2008; 135: 1362-71.

44. Peek GJ, Mugford M, Tiruvoipati R, Wilson A, Allen E, Thalanany MM, et al. Efficacy and economic assessment of conventional ventilatory support versus extracorporeal membrane oxygenation for severe adult respiratory failure (CESAR): a multicentre randomised controlled trial. Lancet 2009; 374: 1351-63. 